\title{
Sonic boom mitigation via shape optimization using an adjoint method and application to a supersonic fighter aircraft
}

\author{
Kurt Maute* — Charbel Farhat** — Brian Argrow* \\ Melike Nikbay***
}

* Department of Aerospace Engineering Sciences, University of Colorado Boulder, CO 80309, USA

\{kurt.maute,brian.argrow\}@colorado.edu

** Department of Mechanical Engineering and Institute for Computational and Mathematical Engineering, Stanford University, Stanford, CA 94305, USA

cfarhat@stanford.edu

*** Faculty of Aeronautics and Astronautics, Istanbul Technical University Maslak, 34469 Istanbul, Turkey

nikbay@itu.edu.tr

ABSTRACT. This paper describes a computational method for the analysis and mitigation via shape optimization of the sonic boom associated with supersonic flight. The method combines a CFD approach for determining the near-field pressure field and an acoustic scheme for predicting the initial shock pressure rise at the ground. Two venues are considered for computing the ground signature. The performance of both approaches is evaluated using flight test data of two different configurations of an F5 fighter aircraft.

RÉSUMÉ. Cet article décrit une méthode numérique pour l'analyse et l'atténuation à travers l'optimisation de formes du bang sonique associé aux vols supersoniques. La méthode associe une approche CFD pour la détermination du champ de pression près du corps à un schéma acoustique pour prédire l'accroissement du choc de pression initial au sol. Deux procédés différents sont considérés et comparés pour le calcul de la signature au sol. Les performances de ces deux procédés sont évaluées en utilisant des données de vol obtenues avec deux configurations différentes d'un avion de combat F5.

KEYWORDS: adjoint method, ray tracing, shape optimization, sonic boom.

MOTS-CLÉS : méthode de l'adjoint, ray tracing, optimisation de formes, bang sonique.

DOI:10.3166/REMN.17.217-243 @ 2008 Lavoisier, Paris

REMN - 17/2008. Shape design in aerodynamics, pages 217 to 243 


\section{Introduction}

During the past two decades, simulation-based design methodologies have flourished for a broad range of applications. These methodologies seamlessly connect computer-aided design (CAD) techniques to numerical schemes for solving partial differential equations. They also allow engineers to predict the performance of engineering systems and to manipulate their geometry and material composition. Critical design decisions can be made at early stages in the design process and the number of costly design iterations involving the fabrication of prototypes can be reduced.

To allow for a formal and systematic design procedure, simulation and CAD techniques have been integrated into mathematical design optimization methods such as nonlinear programming and genetic and evolutionary strategies. Initially, this approach was motivated by time savings due to automating standard design problems and by the possibility of explicitly formulating objectives and constraints. However, it has been recently recognized that one of the major advantages of optimizationbased design methodologies is their ability to generate non-intuitive solutions that do not depend on the experience of the engineer. In particular, this potential has been demonstrated by topology and shape optimization methods for complex single- and multi-physics applications such as the design of mechanisms for microelectromechanical systems and multi-functional materials (Sigmund, 2001; Pajot et al., 2006; Rupp et al., 2007). The complexity of these example applications stems from the large number of candidate designs and/or the coupling of multiple physical fields. Mathematical optimization methods do not only allow for a systematic exploration of the resulting design spaces but are also able to find solutions to seemingly irresolvable, contradictory problems such as micro-structured materials with negative Poisson ratios and refraction indices.

In aerospace engineering, a similar "irresolvable" problem is concerned with the elimination or reduction of the sonic boom caused by an aircraft at supersonic speed. The very moment Chuck Yeager broke the sound barrier in 1947, the question was raised whether or not it is possible to fly supersonic without generating a sonic boom at the ground. However, already in 1955 Busemann showed that eliminating the sonic boom is impossible due to an inescapable lift contribution. Thus, the question remained how to reduce the sonic boom by optimally shaping the aircraft and positioning the propulsion system. To understand the complexity of this problem, one needs to consider the phenomena involved in the generation and propagation of the acoustic signature of an aircraft. As shown in \{Figure 1 a moving body causes a pressure disturbance along its surface at flight altitude. While the near-field pressure disturbance is propagated down to the ground, shocks coalesce forming typically a two-shock signature, such as an $\mathrm{N}$-wave, with a front and a rear shock. The strength of the sonic boom at the ground can be measured, for example, by the total impulse, the initial shock pressure rise (ISPR), and the maximum pressure as illustrated in Figure 2 Consequently, the solution to designing a low-boom aircraft involves predicting the near-field and the far-field pressure fields as well as finding the optimal configuration of the aircraft, including its geometry and propulsion system. 


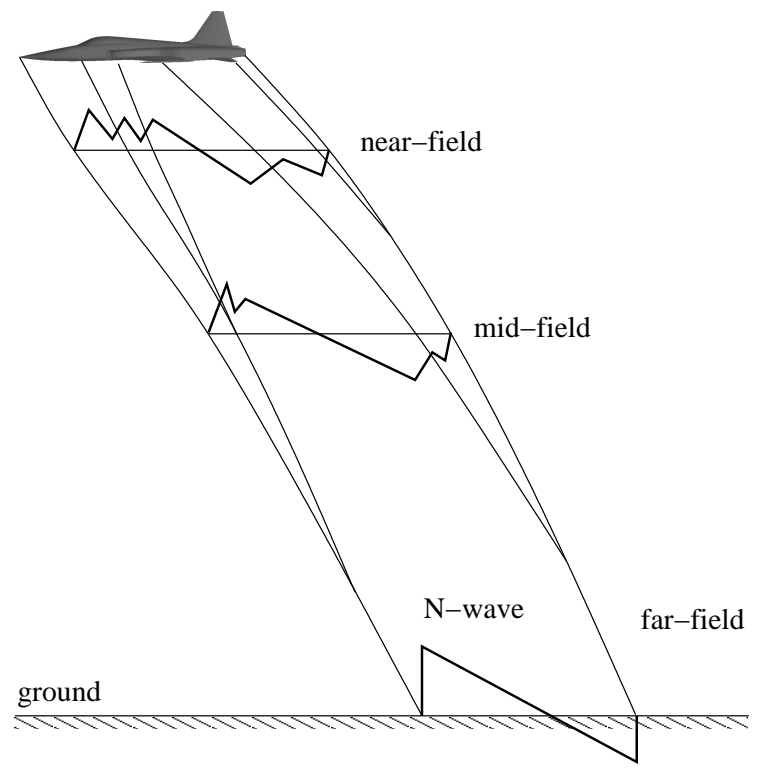

Figure 1. Propagation of pressure disturbance with overpressure signatures at nearfield, mid-field, and at the ground

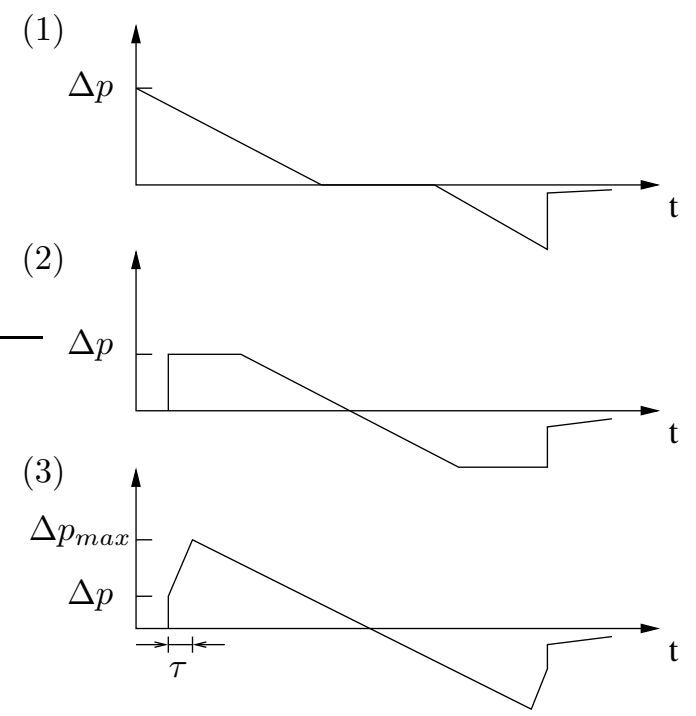

Figure 2. Overpressure signatures: (1) minimum impulse with an ISPR of $\Delta p$, (2) minimum ISPR $\Delta p$, (3) minimum overpressure with ISPR $\Delta p$, followed in a finite rise time $\tau$ by a maximum pressure $\Delta p_{\max }$ 
The near-field pressure can be computed by standard numerical flow solvers, typically based on Euler or Navier-Stokes flow models discretized by finite element or finite volume methods. In theory, the same or similar approaches could be applied for computing the far-field pressure fields. This, however, would require the discretization of the entire space between the aircraft and the ground leading to impractical computational problems. Instead, simplified methods are used to propagate the pressure disturbance from the near-field to the ground. The computationally least expensive and today most commonly used approaches rely on a modified linear theory (MLT) for predicting the ground signature. This theory was introduced by Whitham (1952) and later refined by Lomax (1955) and Walkden (1958). The three-dimensional pressure field is cast into a one-dimensional function, called the $F$-function, describing the wave field of an equivalent body of revolution. The MLT "corrects" the original linearized theory with second-order effects to track the propagation of near-field waves through an isothermal atmosphere into the bow and tail shock of the N-wave asymptotic far-field solution. McLean (1965) introduced a formula to compute the strength of the front and rear shocks for a given $F$-function, reducing the computational burden of propagating the pressure disturbance to building and integrating the $F$-function.

More accurate acoustic propagation methods are not based on the assumption of a two-shock ground signal and model the propagation of the aircraft acoustic signature through the real atmosphere. These methods are typically based on ray-tracing techniques and track individual shocks starting from the near-field pressure distribution or, alternatively, from the corresponding $F$-function. They account for secondorder effects associated with the propagation of weak shocks that accumulate to firstorder shocks and real atmosphere models, such as the US Standard Atmosphere. While more involved and computationally more costly than McLean's approach, these acoustic propagation methods allow for considering arbitrarily shaped waves and finetuning the overall ground signature.

Based on the MLT and the two-shock assumption, lower bounds on the minimum achievable sonic boom were established early on for a given aircraft length, weight, free-stream Mach number, and altitude (George et al., 1969). This approach allows for the optimization of the cross-sectional area distribution of an equivalent body of revolution, separately for one of the performance measures mentioned above (see Figure 2). However, there is no unique relation between the cross-sectional area distribution and the corresponding geometry of an aircraft as both the physical volume and lift distribution contribute to the cross-sectional area distribution of the equivalent body. Thus, the engineer is left with finding the aircraft geometry that matches the optimum $F$-function as well as possible while satisfying structural and aerodynamic constraints. This approach resulted in the low-boom designs of the 1960s and 1970s (Carlson, 1964; Darden, 1977; Carlson et al., 1973).

The most significant shortcomings of the two-step procedure outlined above are two-fold: First, only two-shock ground signatures can be considered. However, these two-shock signatures are developed assuming that all intermediate shocks generated near the vehicle coalesce into the bow and tail shocks before reaching the ground, 
representing the worst-case scenario. More complex ground signatures with multiple shocks typically result in lower total momentum and ISPR. The second shortcoming is due to an inconsistent subdivision of the overall optimization problem into two subproblems. Design constraints cannot be accounted for finding the "optimal" areadistribution. Therefore, the geometry that approximates the "optimum" $F$-function the best is not necessarily the solution of the overall constrained optimization problem.

Motivated by the recently revived interest in supersonic aircraft for civil and military applications (High Speed Civil Transport - Program Review, n.d.; , Quiet Sonic Platform (QSP), n.d.), various optimization approaches have been presented to overcome the shortcomings of the two-step procedure and other methods based on finding an "optimal" equivalent body of revolution (Yamaguchi et al., 1998; Farhat et al., 2002b; Farhat et al., 2004; Farhat et al., 2007; Miles et al., 2002; Farhat et al., 2002a; Nadarajah et al., 2002; Vázquez et al., 2002; Karr et al., 2003; Vázquez et al., 2004; Rallabhandi et al., 2006). All of these alternative approaches operate directly on the true aircraft geometry and integrate a numerical flow solver into a formal optimization method. Most approaches account for additional aerodynamic performance criteria, such as drag and lift, and include these either as part of the objective or as design constraints. With the exception of a few methods relying on genetic algorithms (Yamaguchi et al., 1998; Rallabhandi et al., 2006), the design problem is formulated as a nonlinear program and solved by gradient-based optimization methods. The gradients of the objective and constraints are either evaluated by the direct approach (Farhat et al., 2002b; Farhat et al., 2002a; Farhat et al., 2004) or the adjoint method (Nadarajah et al., 2002; Vázquez et al., 2002).

Significant differences, however, exist with respect to the technique for manipulating the shape and the approach for evaluating the strength of the sonic boom at the ground. Shape variations are described either by a CAD-based approach or by varying directly the position of the vertices on the surface in the computational mesh. The latter approach is often labeled CAD-free. CAD-based techniques typically require a direct link between the original CAD model and the computational mesh. Alternatively, a simplified CAD model can be reconstructed based on the computational mesh (Samareh, 1999; Raulli et al., 2002). The main advantages of CADbased techniques are that possible shape variations can be defined by a small number of optimization variables, and tightly controlled to avoid unwanted shapes from being generated. In comparison, CAD-free approaches allow for a larger variety of shape changes at the cost of lesser shape control and a larger number of optimization variables (Jameson, 1997; Jameson et al., 1998). In addition, varying directly the vertices in the computational mesh limits the magnitude of shape changes as infeasible mesh distortions are likely to happen. Studies on shape optimization for lowboom design have used either a CAD-based approach (Farhat et al., 2002b; Farhat et al., 2002a; Farhat et al., 2004; Farhat et al., 2007) or a CAD-free technique (Nadarajah et al., 2002; Vázquez et al., 2002; Vázquez et al., 2004; Vázquez et al., 2006) but have not directly compared these approaches for the same design problem. Thus, it is not yet clear whether a CAD-based or CAD-free approach is more advantageous for lowboom design. 
A variety of approaches are available for measuring the strength of the sonic boom. On the other hand, only a few optimization methods that evaluate the entire ground signature using a ray-tracing algorithm (Rallabhandi et al., 2006) or that approximate some characteristics of the ground signature such as the ISPR by the MLT (Farhat et al., 2002b; Miles et al., 2002; Farhat et al., 2002a; Farhat et al., 2004; Farhat et al., 2007) have been developed. The major challenge with such approaches is the integration of the ground signature analysis method into the optimization scheme, in particular when gradients are required. Rallabhandi and Mavris (2006) have alleviated these challenges by embedding a ray-tracing method into a genetic algorithm. Farhat et al. (2002b, 2004, 2007) have evaluated the ISPR using McLean's formula (McLean, 1965) and have presented a direct method for evaluating the gradients of the ISPR with respect to the shape parameters. All other published optimization methods construct a cost function that is solely based on the near-field pressure and its spatial derivatives. Nadarajah et al. (2002) have sought to match an "ideal" near-field pressure distribution while also considering the aerodynamic drag in the objective function. Vazquez et al. (2002) have constructed an objective function that includes the norm of the pressure gradients integrated over the reference plane below the aircraft. In addition, the differences between the lift and drag coefficients with respect to target values are minimized. Because all of these studies have considered only one particular formulation of the objective function, it has not yet been established whether mitigating the sonic boom via shape optimization requires evaluating the ground signature using a ray-tracing method or whether using McLean's formula to approximate the ISPR suffices for this purpose.

Hence, this paper focuses on studying the influence of the chosen objective function and shape manipulation technique on the optimal shape for low-boom design. It also presents a general adjoint sensitivity analysis framework for computing the gradients of the sonic boom measure that is independent of the chosen boom analysis method. However, since an indirect near-field-based approximate formulation of the ground signature does not offer any significant advantage over the direct evaluation of the strength of the sonic boom on the ground, such a boom analysis approach is not considered here. Instead, McLean's formula (McLean, 1965) and the ray-tracing algorithm first published in (Hayes et al., 1968) are considered for this purpose. The accuracy of both of these two approaches is assessed using ground signature test data obtained for one original F5 fighter configuration and one that was modified to reduce the ISPR and achieve a flattop ground signature (Pawlowski et al., 2004). This test data is also used to compare the results generated by the various optimization approaches outlined above.

The remainder of this paper is organized as follows. A brief overview of the sonic boom analysis methods used in this study is given in Section 2 An adjoint formulation of the design sensitivity analysis is described in Section 3 The techniques considered in this work for manipulating shape and shape variations are summarized in Section 4 The optimization results obtained using the various methodologies outlined above are discussed in Section 5 Finally, conclusions are offered in Section 6 


\section{Sonic boom analysis}

Here, two approaches are overviewed for evaluating the strength of the sonic boom at the ground, namely, McLean's simple approximation (McLean, 1965) and a raytracing algorithm implemented in the ARAP code (Hayes et al., 1968). Both approaches operate on the $F$-function:

$$
F(y)=\frac{1}{2 \pi} \int_{0}^{y} \frac{S_{V}^{\prime \prime}(x)+S_{L}^{\prime \prime}(x)}{\sqrt{y-x}} d x
$$

which is defined along an axis whose origin is located at or near the nose of the aircraft and runs parallel to the fuselage (see Figure 3). Here, a / superscript denotes a derivative with respect to the spatial coordinate $x$. In Equation [1, $S_{V}$ denotes the cross-sectional area due to the volume of the equivalent body of revolution and is given by

$$
S_{V}(x)=A(x) \sin \theta .
$$

The cross-sectional area $A(x)$ is generated by the intersection of the aircraft and the planes forming a constant angle $\theta$ with the $x$-axis. With $\alpha$ denoting the angle of attack and $\mu$ the Mach angle, the angle $\theta$ in [2] is defined as follows:

$$
\theta=\mu-\alpha \quad \text { with } \quad \mu=\sin ^{-1} \frac{1}{M_{\infty}}
$$

where $M_{\infty}$ denotes the free-stream Mach number. The cross-sectional area of the equivalent body of revolution due to lift, $S_{L}$, is given by

$$
S_{L}(x)=\frac{\beta}{\rho_{\infty} U_{\infty}^{2}} \int_{0}^{x} l(\xi) d \xi,
$$

where $\beta=\sqrt{M_{\infty}^{2}-1}$ is the Prandtl-Glauert factor and $\rho_{\infty}$ and $U_{\infty}$ are the freestream density and speed, respectively. The lift distribution along the $x$ axis is denoted by $l$ and computed from a CFD approximation of the near-field pressure.

\subsection{McLean's formula}

Assuming that multiple shocks present in the near- or mid-field coalesce before reaching the ground, the sonic boom ISPR on the ground can be approximated by:

$$
\Delta p=\frac{2^{\frac{5}{4}}}{H^{\frac{3}{4}}} \frac{\gamma}{\sqrt{\gamma+1}}\left(M_{\infty}^{2}-1\right)^{\frac{1}{8}} \sqrt{p_{a} p_{g} \int_{0}^{y^{0}} F(y) d y}
$$

where $H$ denotes the flight altitude, $\gamma$ is the ratio of specific heats, $p_{a}$ is the pressure at the specified flight altitude and $p_{g}$ the pressure on the ground. The integral over the $F$-function is evaluated from the nose of the aircraft to the balance point, $y^{0}$, with $F\left(y^{0}\right)=0$, capturing all contributions feeding into the front shock (Whitham, 1952; McLean, 1965). 


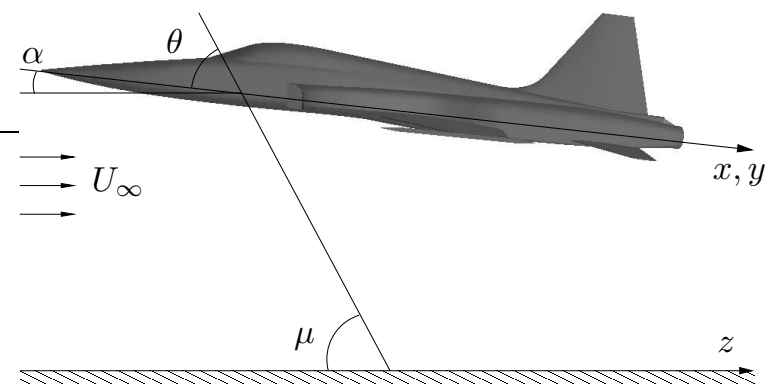

Figure 3. Definition of the $x, y$ and $z$ axes, Mach angle $\mu$, angle of attack $\alpha$, and angle $\theta$

\subsection{Ray-tracing method}

Acoustic ray-tracing methods do not require the assumption on the near- or midfield coalescence, yield more accurate results than McLean's formula and provide the full shape of the ground signature which can then be further processed to extract performance measures such as the ISPR, the maximum overpressure, etc. In this study, the ray-tracing algorithm available in the ARAP code (Hayes et al., 1968) is considered. From the knowledge of the $F$-function, ARAP generates a ground signature $\Delta p(z)$ as a function of $z$. Treating the underlying ray-tracing method as a black box, the propagation of the pressure field can be represented as follows:

$$
\Delta p(z)=\mathcal{M}(y, F(y)) .
$$

The ISPR or the maximum positive overpressure, for example, can then be extracted from $\Delta p(z)$. For optimization purposes, a differentiable approximation of the maximum such as the Kreisselmeier-Steinhauser (KS) function (Kreisselmeier $e t$ $a l ., 1983)$ can be used. If the ground signature $\Delta p(z)$ is given at $N_{g}$ distinct locations $z_{i}, i=1 \ldots N_{g}$, the maximum of $\Delta p\left(z_{i}\right)$ is approximated as follows:

$$
\Delta p_{\max }=\frac{1}{\eta} \ln \left(\sum_{N_{g}} e^{\eta \Delta p\left(z_{i}\right)}\right) \quad \text { with } \eta>1 .
$$

In Section [5] the maximum overpressure approximated by [7] is used as an alternative objective to the ISPR evaluated by McLean's formula.

\subsection{Application to the F5 fighter aircraft}

To illustrate their accuracy properties, the two sonic boom prediction methods outlined above are applied here to the prediction of the ground signatures of two configurations of the F5 fighter aircraft: (a) an original type E configuration (F5-E), and (b) 
a configuration with a reshaped nose section designed specifically to generate a low boom with a flattop ground signature (Pawlowski et al., 2004; Plotkin et al., 2004). In the following, the modified configuration is referred to as the SSBD - that is, the Shaped Sonic Boom Demonstrator. CAD models of the F5-E and SSBD are shown in Figure 4 The ground signatures for both aircraft configurations were measured for $M_{\infty} \approx 1.32$ at the altitude $H=9.75 \mathrm{~km}$.

The underlying CFD technology is built around the finite volume code AERO-F (Farhat et al., 2003) for predicting the near-field pressure distribution and the numerical approximation of the $F$-function described in (Farhat et al., 2002b; Farhat et al., 2004; Farhat et al., 2007). The latter can be used either in conjunction with McLean's formula for predicting the ISPR on the ground or the ARAP code for computing the ground signature.

Modeling in each case only one half of the aircraft and applying symmetry boundary conditions along the symmetry plane, the computational domain for the F5-E contains 1,986,319 tetrahedra and 389, 569 grid points, 98,643 of which are located on the surface of the aircraft. The half-model of the SSBD contains 2, 121, 227 tetrahedra and 417, 017 grid points, 106,375 of which are surface nodes. Estimating the overall weights of the two aircraft configurations (Plotkin et al., 2004), both models were trimmed for a level steady-state flight at $M_{\infty}=1.32$ and $H=9.75 \mathrm{~km}$ by adjusting the angle of attack $\alpha$ so that the lift balances the weight.

The contour plots of the local Mach number are given in Figure 5 for the halfmodels including the symmetry planes. The aerodynamic performance of the trimmed models in terms of lift, drag, and center of pressure measured from the nose of the aircraft are summarized in Table 1 for the full aircraft counterparts. The drag is underestimated as the underlying Euler model accounts only for the wave drag. However, for the purpose of sonic boom analysis and low-boom design, the accuracy of the drag prediction is not particularly important.

The measured and numerically predicted ground signatures and ISPR values are reported in Figure 6 (in English units and in pounds per square feet) and Table 2 respectively. Despite the uncertainty with respect to the exact flight conditions and estimated weights, the flight test and numerical results appear to be in good agreement. For the two-shock signature of the F5-E, both McLean's formula and ARAP predict very accurately the ISPR. As expected, for the multi-shock signature of the SSBD, ARAP predicts a lower ISPR value (13\% in this case) and McLean's formula a higher ISPR value (10\% in this case). Considering the aforementioned data uncertainties, these results not only verify both sonic boom analysis methods but also suggest that McLean's formula leads to a satisfactory approximation of the ISPR even in the case of multiple shocks at the ground. 


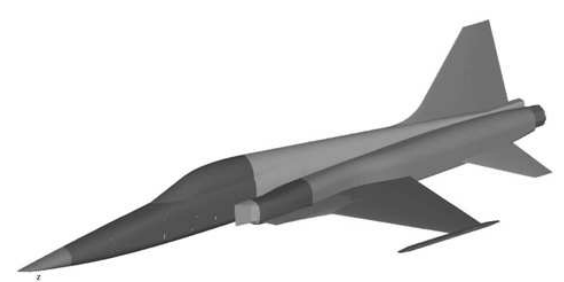

(a) F5-E

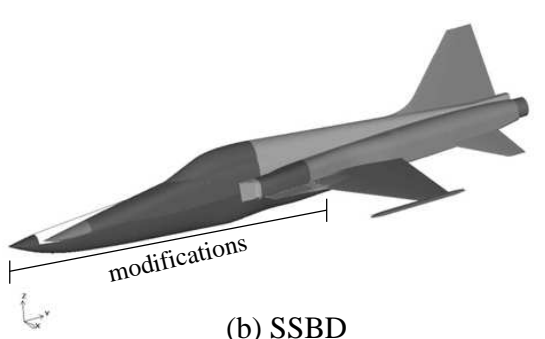

(b) SSBD

Figure 4. CAD models of the F5-E and SSBD designs

Table 1. Lift, drag, center of pressure and angle of attack for the F5-E and SSBD at trimmed state-steady flight

\begin{tabular}{|l|c|c|c|c|}
\hline \hline Configuration & Lift $[k N]$ & Drag $[k N]$ & $x_{c p}[m]$ & $\alpha[\mathrm{deg}]$ \\
\hline F5-E (full model) & 108.85 & 28.18 & 10.30 & 2.05 \\
\hline SSBD (full model) & 108.85 & 30.53 & 10.42 & 2.42 \\
\hline \hline
\end{tabular}

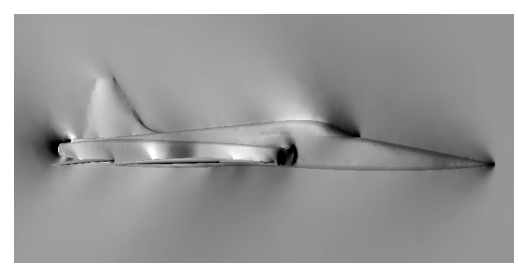

(a) F5-E

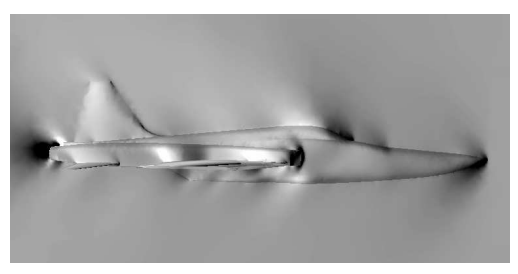

(b) SSBD

Figure 5. Mach number contour plots for the F5-E and SSBD configurations

Table 2. Comparison of the measured and numerically predicted ISPR values for the F5-E and SSBD configurations at trimmed state-steady flight

\begin{tabular}{|l|c|c|c|}
\hline \hline Configuration & $\begin{array}{c}\text { ISPR (measured) } \\
{\left[l b / f t^{2}\right]}\end{array}$ & $\begin{array}{c}\text { ISPR (ARAP) } \\
{\left[l b / f t^{2}\right]}\end{array}$ & $\begin{array}{c}\text { ISPR (McLean) } \\
{\left[l b / f t^{2}\right]}\end{array}$ \\
\hline F5-E & 1.21 & 1.28 & 1.21 \\
\hline SSBD & 0.90 & 0.78 & 0.99 \\
\hline \hline
\end{tabular}




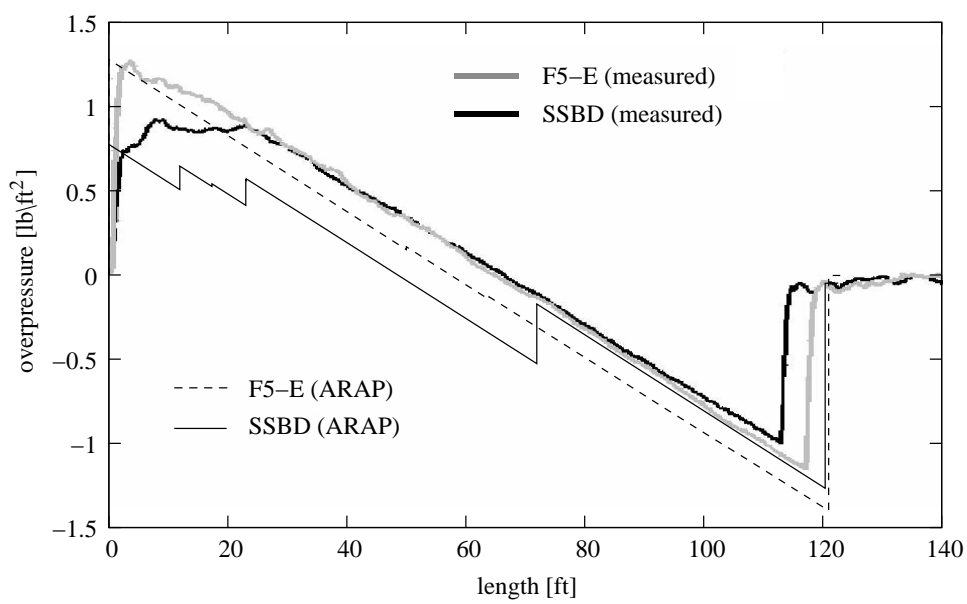

Figure 6. Measured and numerically predicted ground signatures of the F5-E and SSBD configurations

\section{Adjoint sensitivity analysis}

An accurate and efficient method for evaluating the gradients of the objective function and constraints with respect to the optimization variables is a crucial component of a gradient-based optimization scheme. Finite-differencing schemes can be used for low-dimensional problems. They typically diminish the burden of implementation but incur higher computational costs and can lead to inaccurate gradients thereby affecting the convergence of the optimization process. For a large number of optimization variables, such schemes lead to unacceptable computational costs even for small, lowfidelity, CFD models. The authors' experience with various types of flow problems, including those involving coupled fluid-structure interaction (Maute et al., 2001; Maute et al., 2003), is such that they advocate analytical sensitivity analysis methods that feature higher accuracy at lower computational costs. While automatic differentiation tools can alleviate implementation issues, deriving and implementing the analytical sensitivity equations often result in a computationally more efficient segment of code.

In previous work on shape optimization for low-boom design (Farhat et al., 2002b; Farhat et al., 2002a; Farhat et al., 2007), the authors presented a direct formulation for evaluating the gradients of the near-field pressure, the $F$-function and the ISPR evaluated by McLean's formula with respect to optimization variables defining the shape of the aircraft and the free-stream conditions (Mach number and angle of attack). In this paper, an adjoint formulation is introduced allowing the computation of the gradients of the entire ground signature and those of McLean's ISPR approximation with respect to a large number of optimization variables. To this effect and in order to keep the presentation as short as possible, a generic optimization criterion $q$ representing 
the objective function or a constraint is considered. This criterion depends explicitly on the $F$-function and a set, $s$, of $N_{s}$ optimization variables $s_{1}, s_{2}, \ldots s_{N_{s}}$ :

$$
q=q\left(\boldsymbol{s}, F\left(\boldsymbol{s}, \boldsymbol{p}_{\Gamma}\right)\right) .
$$

The $F$-function, in turn, depends on the geometry of the aircraft, the free-stream Mach number, density, and angle of attack, and the near-field pressure distribution represented by the vector of pressure values, $\boldsymbol{p}_{\Gamma}$, at the vertices on the surface of the aircraft $\Gamma$. Equation [8] can represent both McLean's formula [5] as well as any performance measure extracted from the ground signature computed by a ray-tracing method [6] such as the maximum overpressure [7].

The derivative of the optimization criterion, $q$, with respect to the optimization variable $s_{i}$ at a steady-state flow solution can be expressed as follows:

$$
\frac{d q}{d s_{i}}=\frac{\partial q}{\partial s_{i}}+{\frac{\partial q}{\partial p_{\Gamma}}}^{t} \frac{d \boldsymbol{p}_{\Gamma}}{d s_{i}}
$$

where the superscript ${ }^{t}$ denotes the transpose operation. The first term collects all explicit dependencies of $q$ and $F$ on optimization parameters such as the shape of aircraft and the free-stream conditions. These explicit dependencies include the area distribution of the equivalent body of revolution $A(x)$ in the volume contribution $S_{V}$ [2] and its spatial derivatives, as well as the scaling factors in the lift contribution $S_{L}(x)$ [4] and in McLean's formula [5]. The only component that depends on the near-field pressure and the fluid state is the lift distribution $l(x)$ in the lift contribution $S_{L}$ to the $F$-function.

Representing symbolically the steady-state conditions of the discrete flow equations by

$$
\boldsymbol{R}(s, \boldsymbol{w}(s))=\mathbf{0},
$$

where $\boldsymbol{w}$ denotes the vector of fluid state variables in conservative form, the derivative of the pressure $\boldsymbol{p}_{\Gamma}$ can be written as follows:

$$
\frac{d \boldsymbol{p}_{\Gamma}}{d s_{i}}=\frac{\partial \boldsymbol{p}_{\Gamma}}{\partial \boldsymbol{w}} \frac{d \boldsymbol{w}}{d s_{i}}
$$

with

$$
\frac{d \boldsymbol{w}}{d s_{i}}=-\left[\frac{\partial \boldsymbol{R}}{\partial \boldsymbol{w}}\right]^{-1} \frac{\partial \boldsymbol{R}}{\partial s_{i}}
$$

Substituting the expressions for the derivatives of the flow state [12] and for the pressure derivatives [11] into the derivative of the design criterion [9] yields the following equation:

$$
\frac{d q}{d s_{i}}=\frac{\partial q}{\partial s_{i}}-{\frac{\partial q}{\partial p_{\Gamma}}}^{t} \frac{\partial \boldsymbol{p}_{\Gamma}}{\partial \boldsymbol{w}}\left[\frac{\partial \boldsymbol{R}}{\partial \boldsymbol{w}}\right]^{-1} \frac{\partial \boldsymbol{R}}{\partial s_{i}}
$$


The adjoint of the near-field pressure field $\boldsymbol{a}$ is then computed by solving once:

$$
\left[\frac{\partial \boldsymbol{R}}{\partial \boldsymbol{w}}\right]^{t} \boldsymbol{a}={\frac{\partial \boldsymbol{p}_{\Gamma}}{\partial \boldsymbol{w}}}^{t} \frac{\partial q}{\partial \boldsymbol{p}_{\Gamma}}
$$

and then evaluating the remainder of [13] for each optimization variable $s_{i}$ as follows:

$$
\frac{d q}{d s_{i}}=\frac{\partial q}{\partial s_{i}}-\boldsymbol{a}^{t} \frac{\partial \boldsymbol{R}}{\partial s_{i}}
$$

The main advantage of the adjoint approach is that a linear system of equations involving the flux Jacobian is solved only once. In contrast, the direct approach described in (Farhat et al., 2002b; Farhat et al., 2002a; Farhat et al., 2007) requires as many solutions of a linear system of the same size as [14] as there are optimization variables.

The partial derivatives in [14 and [15] can be either evaluated based on the analytically derived discretized fluid equations or by finite differencing. In this work, all partial derivatives are evaluated analytically, with the exception of the derivatives of the sonic boom measure using the ARAP ray-tracing method. The gradients of the $F$-function and McLean's formula with respect to the optimization variables and pressure gradients can be derived analytically (Farhat et al., 2002b; Farhat et al., 2007). Treating the ray-tracing method as a black-box tool requires computing the derivatives $\partial q / \partial \boldsymbol{p}_{\Gamma}$ in [14] and $\partial q / \partial s_{i}$ in [15] by finite differencing. The evaluation of the ground signature, $\Delta p(z)$, by ARAP only depends on the length of the equivalent body of revolution, $y$, and the $F$-function. For both quantities, one can compute the derivatives analytically (Farhat et al., 2002b; Farhat et al., 2007). A numerically efficient finite difference scheme for any $q=q(\Delta p)$ can be constructed by expressing $\Delta p$ as $\Delta p\left(y(\boldsymbol{s}), F\left(\boldsymbol{s}, \boldsymbol{p}_{\Gamma}\right)\right)$ and decomposing $\partial q / \partial s_{i}$ and $\partial q / \partial \boldsymbol{p}_{\Gamma}$ into contributions with respect to the geometry, $y$ and the $F$-function as follows:

$$
\frac{\partial q}{\partial s_{i}}=\frac{\partial q}{\partial(\Delta p)}\left(\frac{\partial(\Delta p)}{\partial y} \frac{\partial y}{\partial s_{i}}+\frac{\partial(\Delta p)}{\partial F} \frac{\partial F}{\partial s_{i}}\right)
$$

and

$$
\frac{\partial q}{\partial \boldsymbol{p}_{\Gamma}}=\frac{\partial q}{\partial(\Delta p)} \frac{\partial(\Delta p)}{\partial F} \frac{\partial F}{\partial \boldsymbol{p}_{\Gamma}} .
$$

The directional derivatives involving the ground signatures are approximated by a central difference scheme with a perturbation size $\epsilon$ :

$$
\frac{\partial(\Delta p)}{\partial F} \frac{\partial F}{\partial \boldsymbol{p}_{\Gamma}} \approx \frac{\Delta p\left(y, F^{p+}\right)-\Delta p\left(y, F^{p-}\right)}{2 \epsilon}
$$

where

$$
F^{p+}=F+\epsilon \frac{\partial F}{\partial \boldsymbol{p}_{\Gamma}} \quad F^{p-}=F-\epsilon \frac{\partial F}{\partial \boldsymbol{p}_{\Gamma}}
$$


and

$$
\left(\frac{\partial(\Delta p)}{\partial y} \frac{\partial y}{\partial s_{i}}+\frac{\partial(\Delta p)}{\partial F} \frac{\partial F}{\partial s_{i}}\right) \approx \frac{\Delta p\left(y^{s+}, F^{s+}\right)-\Delta p\left(y^{s-}, F^{s-}\right)}{2 \epsilon}
$$

with

$$
\begin{array}{ll}
y^{s+}=y+\epsilon \frac{\partial y}{\partial s_{i}} & y^{s-}=y-\epsilon \frac{\partial y}{\partial s_{i}} \\
F^{s+}=F+\epsilon \frac{\partial F}{\partial s_{i}} & F^{s-}=F-\epsilon \frac{\partial F}{\partial s_{i}}
\end{array}
$$

The finite difference approach described above requires two ARAP evaluations for the $\pm \epsilon$ perturbations of the pressure values of each vertex on the surface of the aircraft and for each optimization variable. For applications such as those considered in this paper, the runtime of ARAP is typically in the order of seconds CPU time. The aforementioned partial derivatives are computed only once per iteration of the optimization process. The above finite difference scheme is easily parallelized. For all these reasons, the computational costs associated with this selective finite difference scheme amount to an insignificant overhead when compared to those of the overall optimization process.

As for the derivative of the sonic boom measure, the derivatives of the aerodynamic force and moment criteria such as the drag, lift, and pitch moment can be evaluated by the adjoint approach. Computing the partial derivatives of these criteria is less involved as they only depend on the integration of a function that explicitly depends on the vertex pressures over the surface of the aircraft. In this work, the lift, drag, and pitch moment are considered as constraints. Their derivatives are evaluated by the adjoint method based on analytically derived expressions for the partial derivatives. The reader is referred to Maute et al. (2003) for details about the formulation and implementation of the adjoint sensitivity analysis for aerodynamic force and moment criteria.

\section{Approaches for handling shape variations}

Here, a CAD-based method and a CAD-free approach for describing shape changes for low boom design optimization are compared. In both cases, it is assumed that the optimization process starts from a well-defined initial design and the initial shape is defined through the position of the vertices of the surface fluid mesh, $\boldsymbol{x}_{\Gamma}^{0}$. Even for the CAD-based approach, the authors adopt this problem setup in this work as it does not require defining shape variations in the original CAD model, which can be a complex proposition.

In general, parametric shape variations techniques define shape changes via the motion of the vertices on the surface of the aircraft as a function of the optimization variables $s$.

$$
\boldsymbol{x}_{\Gamma}=\boldsymbol{x}_{\Gamma}^{0}+\Delta \boldsymbol{x}_{\Gamma}(\boldsymbol{s}) .
$$


Both of the CAD-based and CAD-free methods considered here are based on a linear form of $\Delta \boldsymbol{x}\left(s_{i}\right)$ :

$$
\Delta \boldsymbol{x}_{\Gamma}(\boldsymbol{s})=\sum_{N_{s}} \Delta \boldsymbol{x}_{\Gamma, i} s_{i}
$$

where $\Delta \boldsymbol{x}_{\Gamma, i}$ represents a basis vector of the shape change and the optimization variable $s_{i}$ defines the magnitude of the shape change. The main difference between the CAD-based and CAD-free methods considered herein is the approach for constructing the basis vectors $\Delta \boldsymbol{x}_{\Gamma, i}$. In both cases, the shape changes are only defined on the surface $\Gamma$ of the aircraft. However, to allow for large shape changes, the overall volume mesh needs to be updated as the positions of the surface vertices change. For this purpose, a robust and efficient mesh updating strategy developed by Farhat and co-workers (Farhat et al., 2002; Degand et al., 2002) for the numerical simulation of fluid-structure interaction problems is adopted. More specifically, the motion $\Delta \boldsymbol{x}_{\Omega}$ of the vertices within the computational fluid domain $\Omega$ is modeled by the deformation of a fictitious structure subject to prescribed displacements $\Delta \boldsymbol{x}_{\Gamma}$ on the surface $\Gamma$. In matrix format, the mesh updating procedure can be described as the solution of the following problem:

$$
\boldsymbol{K}_{\Omega \Omega} \Delta \boldsymbol{x}_{\Omega}=-\boldsymbol{K}_{\Omega \Gamma} \Delta \boldsymbol{x}_{\Gamma},
$$

where $\boldsymbol{K}_{\Omega \Omega}$ and $\boldsymbol{K}_{\Omega \Gamma}$ are the fictitious stiffness matrices associated with the fluid mesh and constructed using an improved torsional spring approach to prevent crossovers of fluid cells during the update (Farhat et al., 2002; Degand et al., 2002). In general, there is no need to solve the fictitious deformation problem [24] with high accuracy. Usually, a few iterations of a preconditioned conjugate gradient algorithm suffice to update the mesh positions.

For the adjoint sensitivity analysis, equation [15] calls for the evaluation of the derivatives of the flow residual vector with respect to the optimization variables, $\partial \boldsymbol{R} / \partial s_{i}$. As the flow residual depends explicitly on $\boldsymbol{x}_{\Gamma}$ and $\boldsymbol{x}_{\Omega}$, the derivative of the position of the vertices with respect to the shape optimization variables needs to be computed. Differentiating the generic shape variation Equations [22] and [23], the derivative of the position of the vertices on the surface $\Gamma$ is:

$$
\frac{\partial \boldsymbol{x}_{\Gamma}}{\partial s_{i}}=\Delta \boldsymbol{x}_{\Gamma, i} .
$$

Differentiating the mesh motion Equation [24], the derivative of the position of the vertices in the interior of the fluid domain can be computed by solving:

$$
\boldsymbol{K}_{\Omega \Omega} \frac{\partial \boldsymbol{x}_{\Omega}}{\partial s_{i}}=-\boldsymbol{K}_{\Omega \Gamma} \frac{\partial \boldsymbol{x}_{\Gamma}}{\partial s_{i}} .
$$

\subsection{CAD-based shape variation of triangulated surfaces}

In order to define shape variations using CAD interpolation schemes such as splines and Coons patches, a simple CAD model enclosing the segments of the tri- 


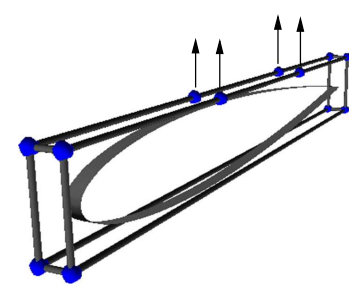

(a) design element model

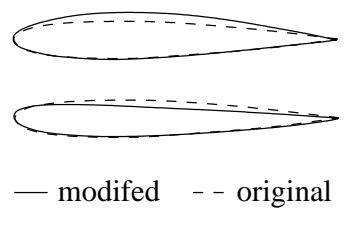

(b) shape variation

Figure 7. CAD-based definition of the shape variations based on the design element concept illustrated with a wing segment

angulated surfaces whose shape is to be varied can be constructed. This is illustrated in Figure 7h using an academic example. Following a design element concept (Imam, 1982; Bletzinger et al., 1991), the CAD model consists of control nodes, edge curves and surface patches that form volume design elements. The position of a vertex $\boldsymbol{x}_{j}$ of the triangulated surface can be uniquely defined by the local coordinate $\boldsymbol{\xi}_{j}$ of the enclosing volume design element:

$$
\boldsymbol{x}_{j}=\sum_{N_{c}} \boldsymbol{N}_{c}\left(\boldsymbol{\xi}_{j}\right) \boldsymbol{x}_{c}
$$

where the matrix $\boldsymbol{N}_{c}$ collects the coefficients of all edge, surface, and volume interpolations and $\boldsymbol{x}_{c}$ are the positions of the $N_{c}$ control nodes of the volume design element. The motion of the control nodes can be defined as a linear function of the optimization variables:

$$
\Delta \boldsymbol{x}_{c}=\sum_{N_{s}} \Delta \boldsymbol{x}_{c, i} s_{i}
$$

where $\Delta \boldsymbol{x}_{c, i}$ denotes the contribution of the $i^{t h}$ optimization variable to the change in the position of the control node $\Delta \boldsymbol{x}_{c}$. Substituting [28] into [27] and comparing the resulting expression with the generic shape variation approach [22] and [23] shows that in the design element method, the components of the basis vector $\Delta \boldsymbol{x}_{\Gamma, i}$ associated with the $j^{\text {th }}$ surface vertex are constructed as follows:

$$
\left(\Delta \boldsymbol{x}_{\Gamma, i}\right)_{j}=\sum_{N_{c}} \boldsymbol{N}_{c}\left(\boldsymbol{\xi}_{j}\right) \Delta \boldsymbol{x}_{c, i} .
$$

The above approach can easily be extended to nonlinear functions linking the optimization variables to the motion of the control nodes. In Figure $7 \mathrm{~b}$, the shape changes due to vertical upward and downward motion of the internal control nodes of the upper Bézier splines are shown. For further details, the reader is referred to (Raulli et al., 2002). 


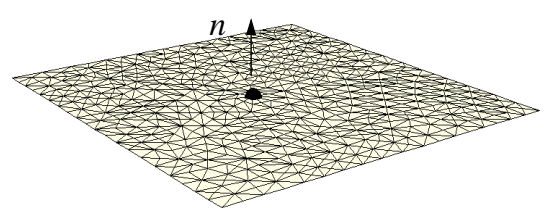

(a) selected vertex

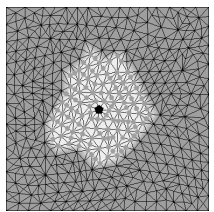

(b) stencil

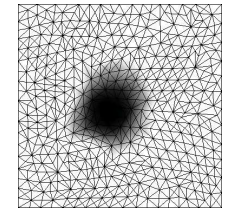

(c) scaling field

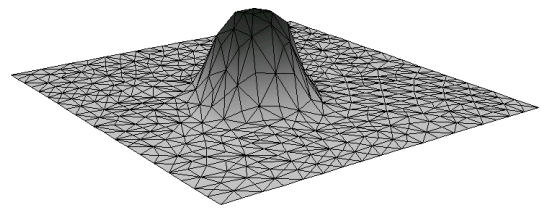

(c) shape variation

Figure 8. $C A D$-free shape variation technique

\section{2. $C A D$-free parametric shape variation}

To allow for a maximum number of possible shape changes, one can treat the motion of each vertex normal to the surface $\Gamma$ as an independent optimization variable. In this case, the basis vector $\Delta \boldsymbol{x}_{\Gamma, i}$ is simply the normal vector $\boldsymbol{n}$ to the surface at $\boldsymbol{x}_{\Gamma}$. However, this simplistic approach results in an ill-posed optimization problem as the discretized fluid model cannot resolve all possible shape variations (Jameson, 1997; Jameson et al., 1998). The shape variations need to be sufficiently smooth with respect to the spatial resolution of the fluid mesh.

Various schemes have been proposed for smoothing the shape variations generated by treating the motion of each vertex in normal direction as an independent optimization variable (Jameson, 1997; Jameson et al., 1998; Vázquez et al., 2002). However, depending on the mesh-updating scheme, this approach may either lead to significant computational costs for computing the mesh motion derivatives or require approximating the mesh motion derivatives. Using the mesh motion scheme described above, for example, the linear system [26] needs to be solved as many times as there are vertices on the surface $\Gamma$. In the case of the F5-E model, there are 98,643 surface vertices leading to unacceptable computational costs. These computational costs can be eliminated, for example, by neglecting the mesh motion derivatives in the interior of the computational domain - that is, by setting $\partial \boldsymbol{x}_{\Omega} / \partial s_{i}=\mathbf{0}-$ at the risk of calculating inaccurate sensitivities and possibly deteriorating the convergence of the overall optimization process.

To overcome the shortcomings of treating the motion of every surface vertex as an independent optimization variable, the following modified technique is suggested and illustrated in Figure 8, (a) select $N_{s}$ surface vertices $\boldsymbol{x}_{i}^{0}$ where $N_{s}$ is typically smaller 
than the total number of surface vertices. The surface vertices can be chosen to obtain either a uniform distribution or one with concentrations in certain areas. (b) Select a stencil that defines a set of nodes $\boldsymbol{x}_{i}^{j}$ in the vicinity of the selected vertex $\boldsymbol{x}_{i}^{0}$. These nodes will move along with $\boldsymbol{x}_{i}^{0}$. The vertex sets should have a sufficient overlap. (c) For each vertex set, construct a smooth scaling field $\kappa_{i}^{j}$ assigning the center vertex $\boldsymbol{x}_{i}^{0}$ the scaling factor $\kappa_{i}^{0}=1$ and the vertices on the stencil boundary $\boldsymbol{x}_{i}^{s b}$ the factor $\kappa_{i}^{s b}=0$. For example, a smooth scaling field can be obtained as the solution of the Laplace equation with Dirichlet boundary conditions $\kappa_{i}^{0}=1$ and $\kappa_{i}^{s b}=0$. (d) Build the components of the basis vector $\Delta \boldsymbol{x}_{\Gamma, i}$ associated with the $j^{\text {th }}$ surface vertex in the $i^{\text {th }}$ vertex set as follows:

$$
\left(\Delta \boldsymbol{x}_{\Gamma, i}\right)_{j}=\kappa_{i}^{j} \boldsymbol{n}_{j}
$$

where $\boldsymbol{n}_{j}$ is the normal vector of the surface $\Gamma$ at the $j^{\text {th }}$ vertex. For vertices located on a symmetry plane, the motion of these vertices is restricted to in-plane directions.

Using the CAD-free scheme described above, the smoothness of the shape changes can be increased by increasing the number of optimization variables and the size of the stencil defining the vertex sets.

\section{Application to the shape optimization of an F5 aircraft for sonic boom mitigation}

To illustrate their applicability and performance, the sonic boom analysis method, adjoint sensitivity analysis approach, and both shape variation techniques described in the previous sections are applied here to produce an alternative SSBD design of the F5-E aircraft. The shape variations are restricted to approximately the same surface sections modified in the SSBD design. Design constraints are imposed on the maximum feasible drag, minimum feasible lift, and on the location of the center of pressure. Two formulations of the objective function are compared: (a) MCL: minimize the ISPR predicted by McLean's formula [5], and (b) RTM: minimize the maximum overpressure based on ARAP's prediction of the ground signature and a maximum approximation by the KS function [7].

Furthermore, the influence of the shape variation technique is studied by comparing the results obtained using the CAD-based (CB) and CAD-free (CF) approaches. The design element model is constructed around the same front section of the F5-E that was modified in the SSBD design and is shown in Figure 92. The design model contains three volume Coons elements with Coons patches as surface elements. The edges along the fuselage axis are 4-node cubic Bézier splines. The remaining edges are represented by 2 -node line elements. The volume elements enclose 2, 045 vertices of the computational surface mesh. The motion of the control nodes is coupled resulting in six basis vectors [29] that are depicted in Figure 90. Using the CAD-free approach, the shape variations are defined by 100 (V100) and 1,000 (V1000) optimization variables. The locations of the center vertices $\boldsymbol{x}_{i}^{0}$ are shown in Figure 10 
(a) Design element model

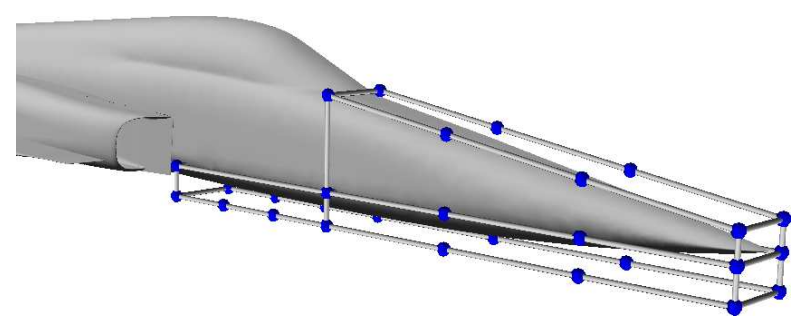

(b) Shape variations associated with optimization variables

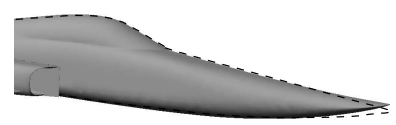

variable 1

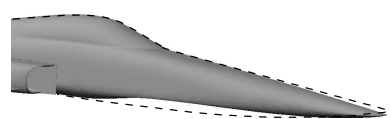

variable 3

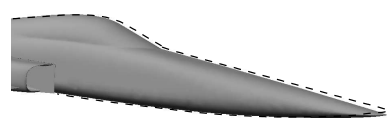

variable 5

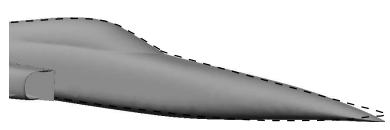

variable 2
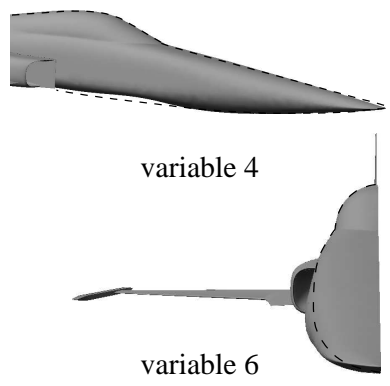

Figure 9. CAD-based definition of shape variations for the F5-E configuration

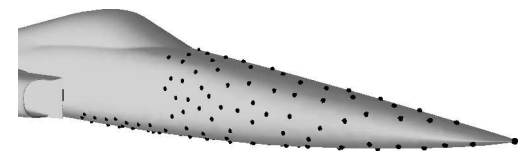

(a) 100 variables

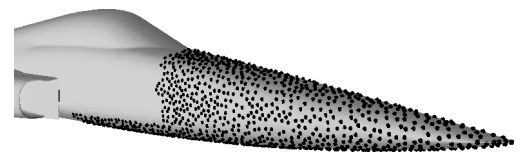

(b) 1000 variables

Figure 10. Locations of the center vertices for the CAD-free approach using 100 and 1000 variables

The two different formulations of the objective function and the CAD-based and CAD-free shape handling methods result in six optimization problems labeled here MCL-CB, MCL-CF-V100, MCL-CF-V1000, RTM-CB, RTM-CF-V100, and 
Table 3. Comparison of the maximum overpressure and ISPR values predicted by ARAP and McLean's formula, respectively, for the optimized F5 configurations

\begin{tabular}{|c|c|c|c|}
\hline \hline & MCL-CB & MCL-CF-V100 & MCL-CF-V1000 \\
\hline$\Delta p_{\max }$ - ARAP $\left[l b / f t^{2}\right]$ & 0.36 & 0.87 & 1.05 \\
\hline ISPR - McLean $\left[l b / f t^{2}\right]$ & 0.64 & 0.88 & 1.04 \\
\hline \hline & RTM-CB & RTM-CF-V100 & RTM-CF-V1000 \\
\hline$\Delta p_{\max }$ - ARAP $\left[l b / f t^{2}\right]$ & 0.64 & 0.84 & 0.77 \\
\hline ISPR - McLean $\left[l b / f t^{2}\right]$ & 0.91 & 0.94 & 1.06 \\
\hline \hline
\end{tabular}

RTM-CF-V1000. For all problems, the resulting nonlinear problem can be formulated as follows:

$$
\begin{array}{rll}
\min _{\boldsymbol{s}} & Z & \\
\text { s.t. } & 1-\frac{D}{D_{\max }} \geq 0 & \text { drag constraint } \\
& \frac{L}{L_{\min }}-1 \geq 0 & \text { lift constraint } \\
& 1-\frac{x_{c p}}{x_{\max }} \geq 0 & \text { center of pressure constraint } \\
& \frac{x_{c p}}{x_{\min }}-1 \geq 0 \quad \text { center of pressure constraint } \\
& \boldsymbol{s} \in \boldsymbol{S}:=\left\{\boldsymbol{s} \in \Re^{N_{s}} \mid \underline{s} \leq \boldsymbol{s} \leq \overline{\boldsymbol{s}}\right\}
\end{array}
$$

where $Z$ is the cost function discussed above, $D$ is the drag, $L$ the lift, and $x_{c p}$ the $\mathrm{x}$-coordinate of the center of pressure. The upper limit of the drag is set to that of the SSBD - that is, $D_{\max }=30.53 \mathrm{kN}$. The lower limit of the lift is set to the weight of the aircraft and therefore $L_{\min }=108.85 \mathrm{kN}$. The center of pressure is allowed to vary between that of the F5-E and that of the SSBD configuration - that is, $x_{\min }=10.30 \mathrm{~m}$ and $x_{\max }=10.42 \mathrm{~m}$. The lower and upper bounds of the optimization variables, $\underline{s}$ and $\bar{s}$ respectively, are set so that the shape changes remain sufficiently small and the mesh update scheme described in Section 4 can generate valid fluid meshes.

In all cases, the nonlinear problem [31] is solved by a sequential quadratic program (Schittkowski, 1985). The optimized shapes and associated Mach contour plots are shown in Figure 11. The ground signatures evaluated by ARAP are plotted in Figure 12 The maximum overpressures based on the ground signatures predicted by ARAP and the ISPR values approximated by McLean's formula are compared in Table 3 The upper part of the table reports on the MCL formulation and the lower part on the RTM problems. In all six cases, only the constraint on the minimum lift turns out to be active.

A dominant design feature characterizing the optimized design and the SSBD is a blunt nose. Depending on the possible design variations, the bluntness is created either by tilting up the nose (MCL-CB and RTM-CB) or by moving the vertices 

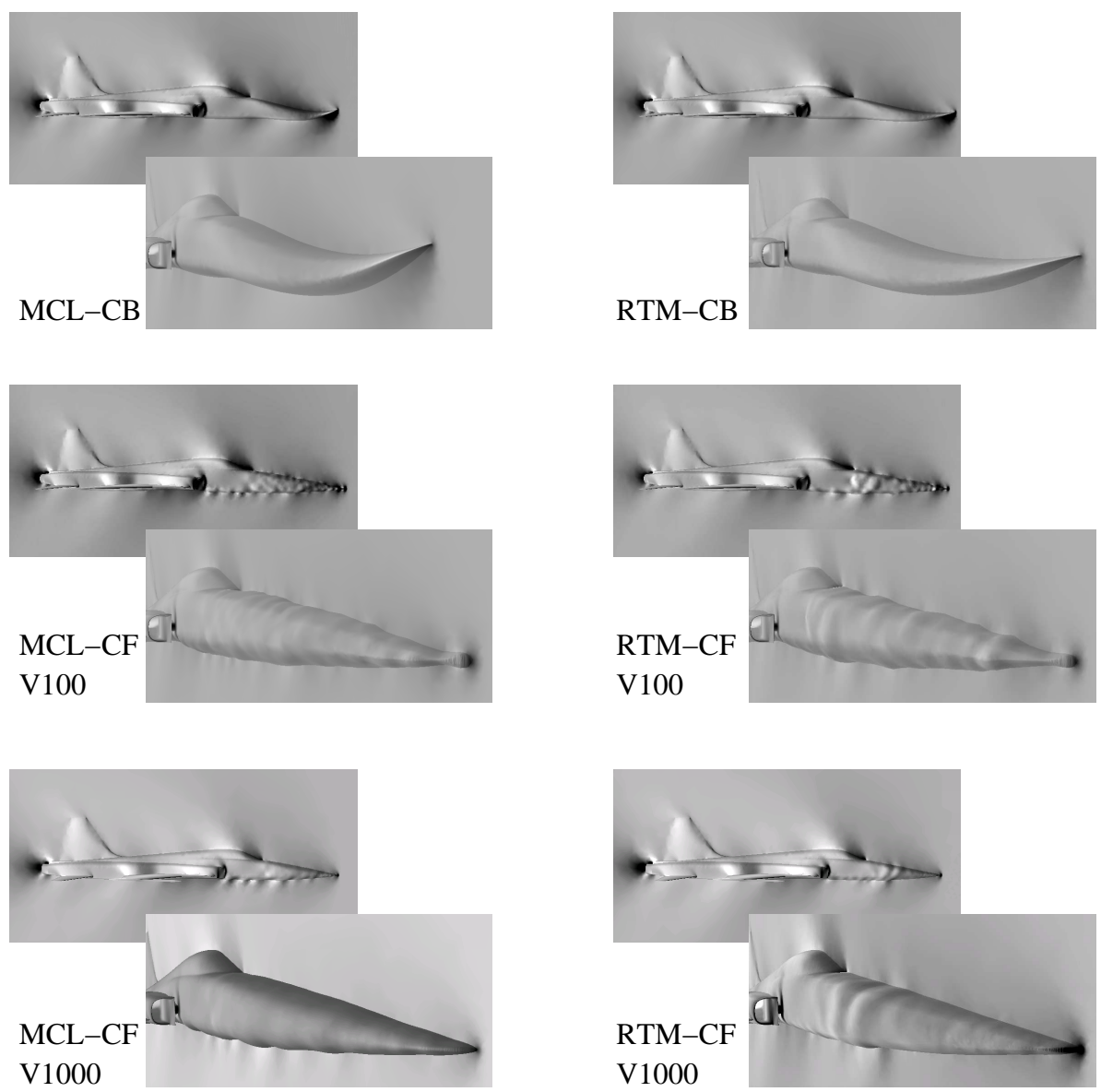

Figure 11. Optimized shapes using the CAD-based and CAD-free approaches and McLean's formula and the maximum overpressure predicted by ARAP as objective functions

on the upper and lower surfaces near the tip upwards and downwards, respectively (MCL-CF-100(0), RTM-CF-100(0)). It is noteworthy that the ground signatures of the optimized designs are remarkably close to the signal produced by the SSBD, as shown in Figure 12 Comparing the shape of the SSBD and optimized designs using the CAD-based approach in Figure 13 shows that the tips of the nose are shaped in a similar fashion creating almost the same local angles of attack. Both optimized designs based on the CAD-free shape variation approach show a remarkably bumpy surface. This effect is particularly pronounced in the cases with 100 design variables. It also exists in the solutions based on 1,000 variables. Therefore, the bumpiness is not solely due to the possible shape changes. As the Mach contour plots for (MCL- 
(a) ground signatures of MCL optimization cases

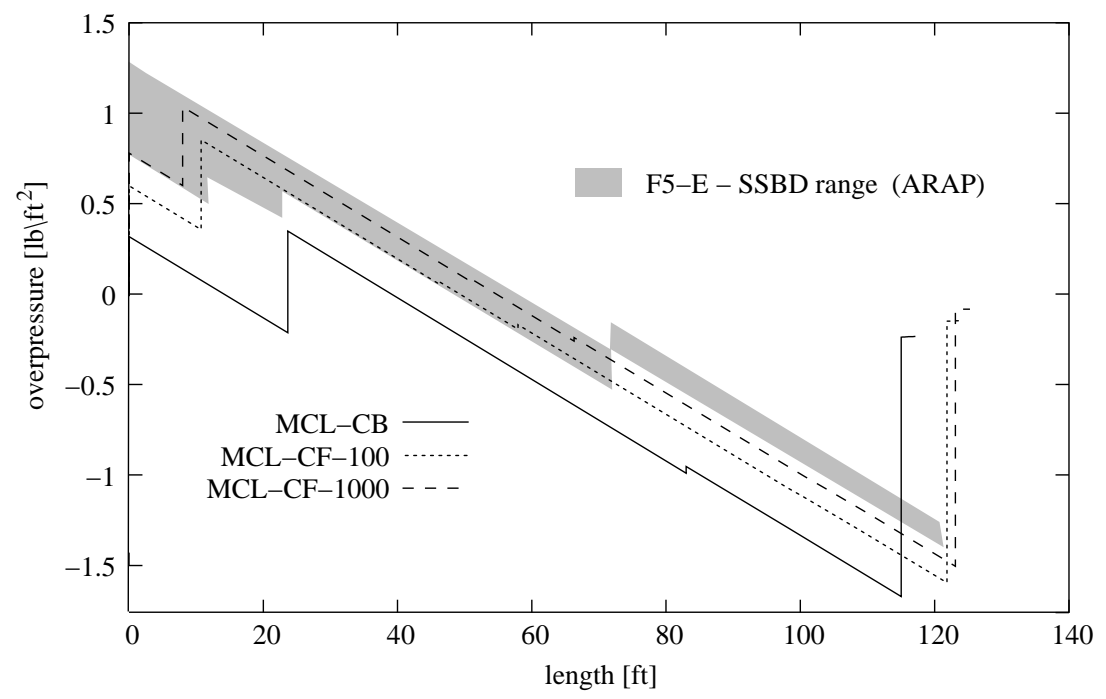

(b) ground signatures of RTM optimization cases

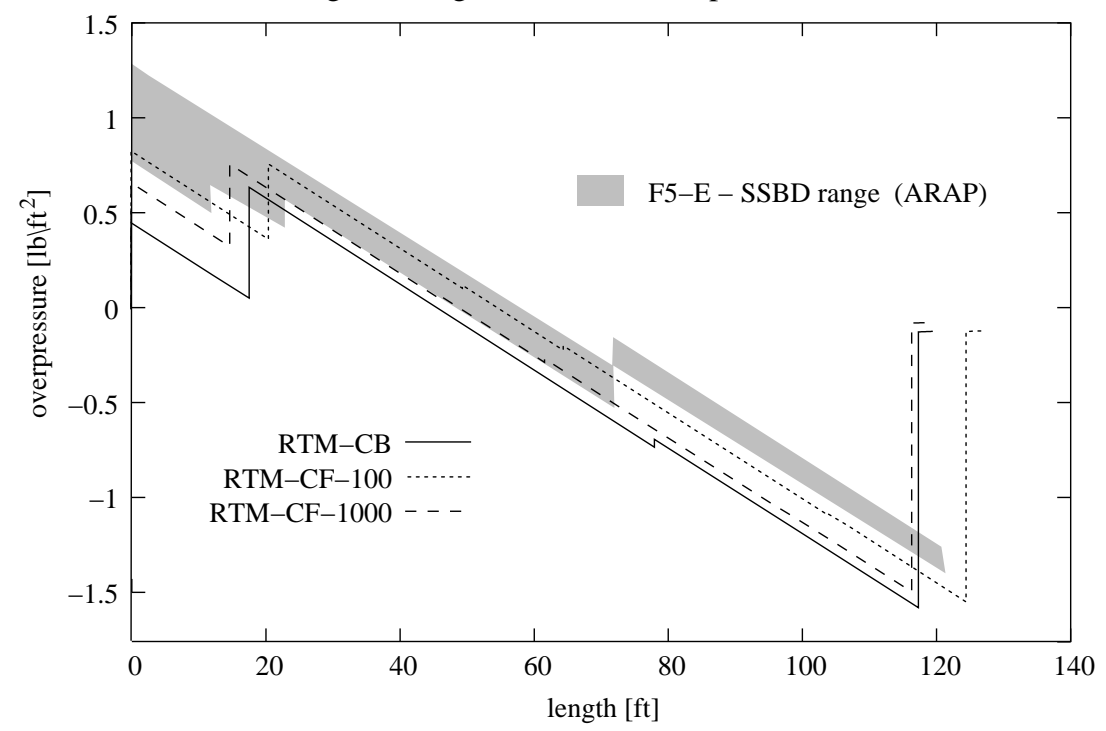

Figure 12. Comparison of the ground signatures predicted by ARAP for the optimized configurations 


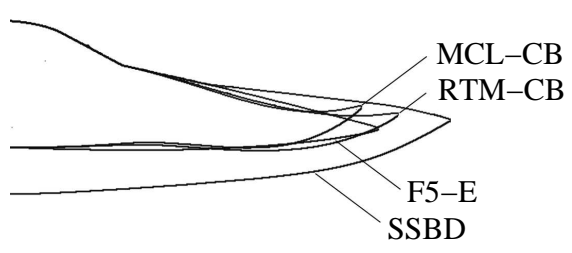

Figure 13. Comparison of the shapes of the the F5-E and SSBD configurations against the optimization results for the CAD-based approach (MCL-CB and RTM-CB)
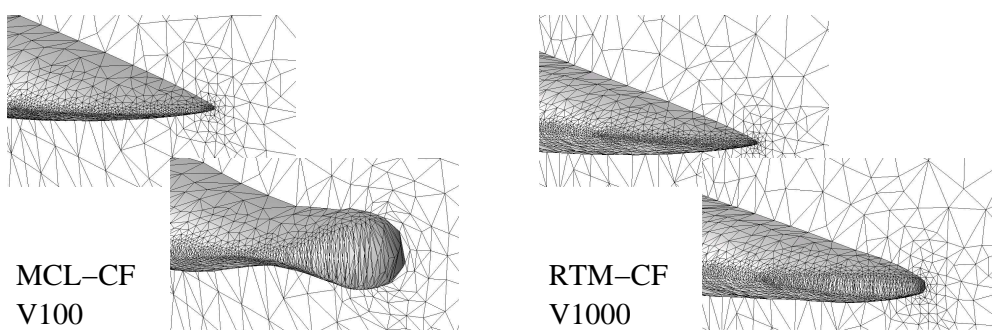

Figure 14. Comparison of the original and optimized shapes at the nose tip for the MCL-CF-V100 and RTM-CF-V1000 cases

CF-100(0) and RTM-CF-100(0)) in Figure 11 show, the bumps initiate waves that prevent the generation of strong two-shock ground signatures.

Optimizing for minimum ISPR using McLean's formula and a CAD-based shape variation technique leads not only to the lowest ISPR but also to the lowest maximum overpressure. Using the same shape variation technique, the resulting design is even better in this case than that obtained by directly minimizing the overpressure.

Using the CAD-free approach, the comparison of the optimization results indicates that the optimum designs for minimum ISPR differ from those for minimum maximum overpressure. However, the optimization results using the CAD-free approach are significantly dominated by the box constraints. To avoid fluid cells collapsing because of large mesh distortions, rather small upper and lower bounds on the optimization variables have to be imposed. The most critical area is at the tip of the aircraft (see Figure 14]. To create a blunt nose, vertices are moved apart in the vertical direction. This creates large mesh distortions and requires limiting the range of the optimization variables. Using the CAD-based approach, such distortion intensive shape variations can be avoided allowing for overall larger shape changes, which lead to larger performance improvements. 


\section{Conclusions}

This paper has been concerned with the shape optimization of supersonic aircraft for low boom design. A sonic boom analysis method based on a CFD approximation of the near-field pressure distribution and two $F$-function based approaches for predicting the strength of the ground signature has been presented. Comparing the simulation results against measured data for an F5-E and the Shaped Sonic Boom Demonstrator (SSBD) F5 aircraft shows that the approximation of the ISPR by McLean's formula and the prediction of the maximum overpressure based on the ARAP acoustic ray-tracing method lead to satisfactory results.

An adjoint formulation of the sonic boom sensitivity analysis was presented. The evaluation of the gradients of McLean's approximation of the ISPR and of the ground signature predicted by ARAP was also discussed. While treating the ray-tracing method as a black-box tool necessitates the partial use of finite difference approximations, the described sensitivity formulations allow for the efficient evaluations of the gradients of the ISPR and maximum overpressure for a large number of optimization variables at acceptable computational costs.

So far, studies on the shape optimization for low boom design have either relied on CAD-based or CAD-free techniques for describing the shape changes during the optimization process. A general framework for both approaches was presented, a CAD-based approach operating on a triangulated surface mesh was discussed, and a CAD-free technique allowing for selected shape variations was introduced.

The developed optimization methodology was applied to the redesign of the F5E configuration allowing for shape changes along the nose section that are similar to those effected on the SSBD. The influence of the choice of representation of the objective function and of the shape variation technique on the optimal design was studied. The ground signatures of the optimized designs are well within the range of the original and the SSBD designs but show an improved performance with respect to ISPR and maximum overpressure. Similar to the SSBD design, the sonic boom is reduced by creating a blunt nose. In particular the optimized designs for the CADbased approach show the same effective design at the tip of the nose as the SSBD.

The obtained results suggest that McLean's formula is well-suited for minimizing both the ISPR and the maximum overpressure. Using the maximum overpressure predicted by ARAP as an objective function seems to result in optimization problems with multiple local minima. While the CAD-based shape variation technique allowed for rather large shape changes with significant boom reduction, major problems with the CAD-free technique were experienced imposing unacceptable mesh distortions at the tip of the aircraft. 


\section{Acknowledgments}

The first author acknowledges the support of the National Science Foundation under grant DMI-0348759. The opinions and conclusions presented in this paper are those of the authors and do not necessarily reflect the views of the sponsoring organization.

\section{References}

Bletzinger K.-U., Kimmich S., Ramm E., “ Efficient Modeling in Shape Optimal Design”, Computing Systems in Engineering, vol. 2, 1991, p. 483-495.

Busemann A., “ The Relation between Minimizing Drag and Noise at Supersonic Speeds", Proceedings of High Speed Aerodynamics, Polytechnic Institute of Brooklyn, 1955, p. 133144.

Carlson H. W., Correlation of Sonic-boom Theory with Wind-tunnel and Flight Measurements, Technical Report, NASA TR R-213, 1964.

Carlson H. W., Barger R. L., Mack R. J., Application of Sonic-boom Minimization Concepts in Supersonic Transport Design, Technical Report, NASA TN D-7218, 1973.

Darden C. M., " Sonic Boom Theory: Its Status in Prediction and Minimization”, J. Aircraft, vol. $129, \mathrm{n}^{\circ} 6,1977$, p. 569-576.

Degand C., Farhat C., “ A Three-dimensional Torsional Spring Analogy Method for Unstructured Dynamic Meshes”, Computers and Structures, vol. 80, n 3-4, 2002, p. 305-316.

Farhat C., Degand C., Koobus B., Lesoinne M., “ Torsional Springs for Two-Dimensional Dynamic Unstructured Fluid Meshes", Computer Methods in Applied Mechanics and Engineering, vol. 163, 1998, p. 231-245.

Farhat C., Argrow B., Nikbay M., Maute K., “A Shape Optimization Methodology with F-Function Lobe Balancing for Mitigating the Sonic Boom”, AIAA 2002-5551, 49th AIAA/ISSMO Symposium on Multidisciplinary Analysis and Optimization, September 4-6, 2002, Atlanta, GA, 2002a.

Farhat C., Geuzaine P., Brown G., “ Application of a Three-Field Nonlinear Fluid-Structure Formulation to the Prediction of the Aeroelastic Parameters of an F-16 Fighter", Computers and Fluids, vol. 32, n ${ }^{\circ}$, 2003, p. 3-29.

Farhat C., Maute K., Argrow B., Nikbay M., “ A Shape Optimization Methodology for Reducing the Sonic Boom Initial Pressure Rise", AIAA 2002-0145, 40th Aerospace Sciences Meeting \& Exhibit, January 14-17, 2002, Reno, NV, 2002b.

Farhat C., Argrow B., Nikbay M., Maute K., " Shape Optimization with F-Function Balancing for Reducing the Sonic Boom Initial Shock Pressure Rise", The International Journal of Aeroacoustics, vol. 3, 2004, p. 361-377.

Farhat C., Maute K., Argrow B., Nikbay M., “ A Shape Optimization Methodology for Reducing the Sonic Boom Initial Pressure Rise”, AIAA J., vol. 45, 2007, p. 1007-1018.

George A., Seebass R., " Sonic Boom Minimization including both Front and Rear Shock Waves”, AIAA J., vol. 10, n 10, 1969, p. 2091-2093.

Hayes W. D., Haefeli R. C., “ The ARAP Sonic Boom Program”, Sonic Boom Workshop, NASA $S P-180,1968$, p. 151-158. 
242 REMN - 17/2008. Shape design in aerodynamics

High Speed Civil Transport - Program Review, Boeing Commercial Airplane Group, P.O. Box 3707, Seattle, WA 98124-2207, n.d.

Imam M. H., “ Three-dimensional Shape Optimization”, International Journal for Numerical Methods in Engineering, vol. 18, 1982, p. 661-673.

Jameson A., Essential Elements of Computational Algorithms for Aerodynamic Analysis and Design, Technical Report, NASA/CR-97-206268, ICASE Report No. 97-68, 1997.

Jameson A., Martinelli L., Pierce N., “ Optimum Aerodynamic Design using the Navier-Stokes Equations", Theoretical and Computational Fluid Dynamics, vol. 10, 1998, p. 213-237.

Karr C., Bowersox R., Singh V., “ Minimization of Sonic Boom on Supersonic Aircraft Using an Evolutionary Algorithm", Proceedings of GECCO, Springer-Verlag Berlin Heidelberg, 2003, p. 2157-2167.

Kreisselmeier G., Steinhauser R., “ Application of Vector Performance Optimization to a Robust Control Loop Design for a Fighter Aircraft”, Int. J. of Control, vol. 37, 1983, p. 251284.

Lomax H., The Wave Drag of Arbitrary Configurations in Linearized Flow as determined by Areas and Forces in Oblique Planes, Technical Report, NACA RM A55A18, 1955.

Maman N., Farhat C., " Matching Fluid and Structure Meshes for Aeroelastic Computations: A Parallel Approach”, Computers and Structures, vol. 54, 1995, p. 779-785.

Maute K., Nikbay M., Farhat C., " Coupled Analytical Sensitivity Analysis and Optimization of Three-Dimensional Nonlinear Aeroelastic Systems”, AIAA Journal, vol. 39, n 11, 2001, p. 2051-2061.

Maute K., Nikbay M., Farhat C., " Sensitivity Analysis and Design Optimization of ThreeDimensional Nonlinear Aeroelastic Systems by the Adjoint Method", International Journal for Numerical Methods in Engineering, vol. 56, $n^{\circ}$ 6, 2003, p. 911-933.

McLean F. E., Some Nonasymptotic Effects on the Sonic Boom of Large Airplanes, Technical Report, NASA TN D-2877, 1965.

Miles R., Martinelli L., Macheret S., Shneider M., Girgis I., Zaidi S., Mansfield D., Siclari M., Smereczniak P., Kashuba R., Vogel P., “ Suppression of Sonic Boom by Dynamic Offbody Energy Addition and Shape Optimization”, AIAA 2002-1500, 40th Aerospace Sciences Meeting \& Exhibit, January 14-17, 2002, Reno, NV, 2002.

Nadarajah S., Jameson A., Alonso J., " Sonic Boom Reduction using an Adjoint Method for Wing-Body Configurations in Supersonci Flows", AIAA 2002-5547, 49th AIAA/ISSMO Symposium on Multidisciplinary Analysis and Optimization, September 4-6, 2002, Atlanta, GA, 2002.

Pajot J., Maute K., Zhang Y., Dunn M., “ Design of Patterned Multilayer Films with Eigenstrains by Topology Optimization”, Journal for Solids and Structures, vol. 43, n 6, 2006, p. $1832-1853$.

Pawlowski J., Graham D., “ Shaped Sonic Boom Demonstration - Program Overview”, http://www.sonicbooms.org, 2004.

Plotkin K., Page J., Graham D., Pawlowski J., Schein D., Coen P., “ Ground Measurements of a Shaped Sonic Boom”, AIAA-2004-2923, 10th AIAA/CEAS Aeroacoustics Conference, May 10-12, 2004, Manchester, Great Britain, 2004.

Quiet Sonic Platform (QSP), http://www.darpa.mil/tto/programs/qsp.html, n.d. 
Rallabhandi S., Mavris D., "Sonic Boom Minimization using Improved Linearized Tools and Probabilistic Propagation”, J. Aircraft, vol. 43, n 6, 2006, p. 1815-1823.

Raulli M., Maute K., " Symbolic Geometric Modeling and Parametrization for Multiphysics Shape Optimization", AIAA 2002-5648, 9th AIAA/ISSMO Symposium on Multidisciplinary Analysis and Optimization, September 4-6, 2002, Atlanta, USA, 2002.

Rupp C., Evgrafov A., Maute K., Dunn M., “Design of Phononic Materials/Structures for Surface Wave Devices Using Topology Optimization”, Structural and Multidisciplinary Optimization, vol. 34, n² 2007, p. 111-122.

Samareh J., " A Survey of Shape Paramterization Techniques", CEAS/AIAA/ICASE/NASA Langley International Forum on Aeroelasticity and Structural Dynamics, June 22-25, 1999, Williamsburg, VA, 1999, p. 333-343.

Schittkowski K., “NLPQL: A FORTRAN Subroutine for Solving Constrained Nonlinear Programming Problems”, Annals of Operations Research, vol. 5, 1985, p. 485-500.

Sigmund O., “ Design of Multiphysics Actuators using Topology Optimization - Part I: Onematerial Structures", Computer Methods in Applied Mechanics and Engineering, vol. 190, $\mathrm{n}^{\circ}$ 49-50, 2001, p. 6577-6604.

Vázquez M., Koobus B., Dervieux A., Aerodynamical and Sonic Boom Optimization of a Supersonic Aircraft, Technical report, INRIA Research Report RR-4520, 2002.

Vázquez M., Koobus B., Dervieux A., “ Multilevel Optimization of a Supersonic Aircraft”, Finite Elements in Analysis and Design, vol. 40, n 15, 2004, p. 2101 - 2124.

Vázquez M., Koobus B., Dervieux A., “ A methodology for the Shape Optimization of Flexible Wings”, Engineering Computations, vol. 23, n 4, 2006, p. 344-367.

Walkden F., " The Shock Pattern of a Wing-body Combination, far from the Flight Path", Aeronautical Quarterly, vol. IX, n², 1958, p. 164-194.

Whitham G., " The Flow Pattern of a Supersonic Projectile”, Commun. Pure Appl. Math., vol. 5, $\mathrm{n}^{\circ} 3,1952$, p. 301-348.

Yamaguchi H., Nakamura Y., “ Optimization of Low Boom Configuration of SST by Genetic Algorithm”, AIAA Paper No. 98-2899, 29th AIAA Fluid Dynamics Conference, June 15-18, 1998, Albuquerque, New Mexico, 1998. 
